\title{
Constant Sign and Nodal Solutions for Nonlinear Robin Equations with Locally Defined Source Term
}

\author{
Nikolaos S. Papageorgiou ${ }^{a}$, Calogero Vetro $^{b}$ and \\ Francesca Vetro ${ }^{c, d}$ \\ ${ }^{a}$ National Technical University, Department of Mathematics \\ Zografou campus, 15780, Athens, Greece \\ ${ }^{b}$ University of Palermo, Department of Mathematics and Computer Science \\ Via Archirafi 34, 90123, Palermo, Italy \\ ${ }^{c}$ Nonlinear Analysis Research Group, Ton Duc Thang University \\ Ho Chi Minh City, Vietnam \\ ${ }^{d}$ Faculty of Mathematics and Statistics, Ton Duc Thang University \\ Ho Chi Minh City, Vietnam \\ E-mail: npapg@math.ntua.gr \\ E-mail: calogero.vetro@unipa.it \\ E-mail(corresp.): francescavetro@tdtu.edu.vn
}

Received August 5, 2019; revised March 20, 2020; accepted March 20, 2020

\begin{abstract}
We consider a parametric Robin problem driven by a nonlinear, nonhomogeneous differential operator which includes as special cases the $p$-Laplacian and the $(p, q)$-Laplacian. The source term is parametric and only locally defined (that is, in a neighborhood of zero). Using suitable cut-off techniques together with variational tools and comparison principles, we show that for all big values of the parameter, the problem has at least three nontrivial smooth solutions, all with sign information (positive, negative and nodal).
\end{abstract}

Keywords: locally defined reaction, nonlinear regularity, nonlinear maximum principle, constant sign and nodal solutions, critical groups.

AMS Subject Classification: 35J20; 35J60.

Copyright (c) 2020 The Author(s). Published by VGTU Press

This is an Open Access article distributed under the terms of the Creative Commons Attribution License (http://creativecommons.org/licenses/by/4.0/), which permits unrestricted use, distribution, and reproduction in any medium, provided the original author and source are credited. 


\section{Introduction}

Let $\Omega \subseteq \mathbb{R}^{N}$ be a bounded domain with a $C^{2}$-boundary $\partial \Omega$. We study the following nonlinear parametric Robin problem

$$
\left\{\begin{array}{c}
-\operatorname{div} a(\nabla u(z))+\xi(z)|u(z)|^{p-2} u(z)=\lambda f(z, u(z)) \text { in } \Omega \\
\frac{\partial u}{\partial n_{a}}+\beta(z)|u|^{p-2} u=0 \text { on } \partial \Omega, \lambda>0,1<p<+\infty
\end{array}\right.
$$

In this problem, the map $a: \mathbb{R}^{N} \rightarrow \mathbb{R}^{N}$ involved in the differential operator of $\left(P_{\lambda}\right)$, is strictly monotone and continuous, hence maximal monotone too. It satisfies certain other growth and regularity conditions, listed in hypotheses $H(a)$ below. These conditions are not restrictive and provide a broad framework in which we can fit many differential operators of interest such as the $p$-Laplacian, the $(p, q)$-Laplacian (that is, the sum of a $p$-Laplacian and of a $q$ Laplacian) and the modified capillary differential operator. We point out that the differential operator is not homogeneous and this is a source of difficulties in the analysis of problem $\left(P_{\lambda}\right)$. The potential function $\xi(\cdot) \in L^{\infty}(\Omega)$ and $\xi(z) \geq 0$ for a.a. $z \in \Omega$. The source term $\lambda f(z, x)$ is parametric with $\lambda>0$ being the parameter and $f(z, x)$ a Carathéodory function (that is, for all $x \in \mathbb{R}$, $z \rightarrow f(z, x)$ is measurable and for a.a. $z \in \Omega, x \rightarrow f(z, x)$ is continuous). The interesting and distinguishing feature of our work is that this source term $f(z, \cdot)$ is only locally defined, that is, we only fix the properties of $f(z, \cdot)$ near zero. Away from that neighborhood of zero, $f(z, \cdot)$ can be arbitrary.

In the boundary condition, $\frac{\partial u}{\partial n_{a}}$ denotes the conormal derivative of $u$ corresponding to the map $a(\cdot)$. This derivative is understood via the nonlinear Green's identity (see Papageorgiou-Rădulescu-Repovš [16], Corollary 1.5.16, p. 34) and when $u \in C^{1}(\bar{\Omega})$, then

$$
\frac{\partial u}{\partial n_{a}}=(a(\nabla u), n)_{\mathbb{R}^{N}}
$$

with $n(\cdot)$ being the outward unit normal on $\partial \Omega$. The boundary coefficient $\beta(\cdot)$ is nonnegative. The case $\beta \equiv 0$ is also included and corresponds to the Neumann problem.

Using suitable cut-off techniques together with variational tools based in the critical point theory, we show that for all $\lambda>0$ big problem $\left(P_{\lambda}\right)$ has at least three nontrivial smooth solutions all with sign information, a positive, a negative and a nodal (sign changing) solutions.

The first to examine parametric elliptic equations with a source term defined only locally, was Wang [20], who studied a semilinear equation driven by the Dirichlet Laplacian. Imposing a local symmetry condition on the source term (it is assumed that it is odd), Wang [20] proves that for every parameter value $\lambda>0$, the problem has a whole sequence $\left\{u_{n}\right\}_{n \geq 1} \subseteq H_{0}^{1}(\Omega) \cap L^{\infty}(\Omega)$ of weak solutions such that $\left\|u_{n}\right\|_{\infty} \rightarrow 0$ as $n \rightarrow+\infty$. The work of Wang [20] was extended by Li-Wang [7] to semilinear Schrödinger equations. Extensions of these results were obtained by Papageorgiou-Vetro-Vetro [18] (semilinear Robin problems), Papageorgiou-Rădulescu-Repovš [15] (nonlinear Robin problems) and Papageorgiou-Rădulescu-Repovš [14] (Dirichlet $(p, 2)$-equations). In all 
the aforementioned works the source term is symmetric near zero and this leads to an application of a version of the symmetric mountain pass theorem, which generates the desired sequence of distinct nodal solutions. In contrast here we do not impose any symmetry condition on $f(z, \cdot)$. Finally we should mention the recent paper of Guarnotta-Marano-Papageorgiou [5]. There the authors also deal with a nonlinear Robin problem driven by a nonhomogeneous differential operator plus an indefinite potential term. However, in [5] the reaction term is nonparametric and this changes the hypotheses on the reaction and consequently the geometry of the problem and the approach used. In [5] it is assumed that the reaction has constant sign near zero (see hypothesis $\left(f_{1}\right)$ ) and this is crucial in the analysis since is the reason for which the reaction is only locally defined. In contrast here we rely on cut-off techniques. Moreover, the asymptotic condition as $x \rightarrow 0$ on the reaction is in our case different and do not require the presence of a concave term near zero (compare $H(f)(i),(i i)$ of this paper with $\left(f_{3}\right),\left(f_{4}\right)$ of [5]). All these facts distinguish our work here from that of [5] and for that reason the tools and techniques are different.

\section{Mathematical background - hypotheses}

Let $l \in C^{1}((0,+\infty))$ with $l(t)>0$ for all $t>0$. We assume the following:

$$
0<\widehat{c} \leq t l^{\prime}(t) / l(t) \leq c_{0} \quad \text { and } \quad c_{1} t^{p-1} \leq l(t) \leq c_{2}\left[t^{s-1}+t^{p-1}\right]
$$

for all $t>0$, with $1 \leq s<p<+\infty, c_{1}, c_{2}>0$.

Then the hypotheses on the map $a(\cdot)$ involved in the definition of the differential operator are the following:

$H(a): a(y)=a_{0}(|y|) y$ for all $y \in \mathbb{R}^{N}$ with $a_{0}(t)>0$ for all $t>0$ and

(i) $a_{0} \in C^{1}((0,+\infty)), t \rightarrow a_{0}(t) t$ is strictly increasing on $(0,+\infty), a_{0}(t) t \rightarrow 0^{+}$ as $t \rightarrow 0^{+}$and $\lim _{t \rightarrow 0^{+}} \frac{a_{0}^{\prime}(t) t}{a_{0}(t)}>-1$;

(ii) there exists $c_{3}>0$ such that $|\nabla a(y)| \leq c_{3} \frac{l(|y|)}{|y|}$ for all $y \in \mathbb{R}^{N} \backslash\{0\}$;

(iii) $(\nabla a(y) \xi, \xi)_{\mathbb{R}^{N}} \geq \frac{l(|y|)}{|y|}|\xi|^{2}$ for all $y \in \mathbb{R}^{N} \backslash\{0\}$, all $\xi \in \mathbb{R}^{N}$;

(iv) if $G_{0}(t)=\int_{0}^{t} a_{0}(s) s d s$ for all $t>0$, then $p G_{0}(t)-a_{0}(t) t^{2} \geq 0$ for all $t>0$ and for some $q \in(1, p)$ we have $\lim _{t \rightarrow 0} \frac{G_{0}(t)}{t^{q}} \leq c^{*}$, some $c^{*}>0$.

Remark 1. Hypotheses $H(a)(i),($ ii $),($ iii $)$ come from the nonlinear regularity theory of Lieberman [8] and the nonlinear maximum principle of Pucci-Serrin [19], while hypothesis $H(a)(i v)$ is motivated by the particular needs of our problem. However, the condition is mild and it is satisfied in all cases of interest.

Clearly $G_{0}(\cdot)$ is strictly increasing and strictly convex. We set $G(y)=$ $G_{0}(|y|)$ for all $y \in \mathbb{R}^{N}$. Then $G(\cdot)$ is convex and differentiable and we have

$$
\nabla G(y)=G_{0}^{\prime}(|y|) \frac{y}{|y|}=a_{0}(|y|) y=a(y) \quad \text { for all } y \in \mathbb{R}^{N} \backslash\{0\} .
$$


Therefore $G(\cdot)$ is the primitive of $a(\cdot)$ and from the convexity of $G(\cdot)$ and since $G(0)=0$, we have

$$
G(y) \leq(a(y), y)_{\mathbb{R}^{N}} \quad \text { for all } y \in \mathbb{R}^{N} .
$$

Hypotheses $H(a)$ lead to the following properties of the map $a(\cdot)$ (see Papageorgiou-Rădulescu [11]).

Lemma 1. If hypotheses $H(a)(i),($ ii $),($ iii $)$ hold, then

(a) $a(\cdot)$ is continuous and strictly monotone (thus maximal monotone too);

(b) $|a(y)| \leq c_{4}\left[|y|^{s-1}+|y|^{p-1}\right]$ for some $c_{4}>0$, all $y \in \mathbb{R}^{N}$;

(c) $(a(y), y)_{\mathbb{R}^{N}} \geq \frac{c_{1}}{p-1}|y|^{p}$ for all $y \in \mathbb{R}^{N}$.

This lemma and (2.1) lead to the following bilateral growth estimates for the primitive $G(\cdot)$.

Corollary 1. If hypotheses $H(a)(i),(i i),($ iii $)$ hold, then

$$
\frac{c_{1}}{p(p-1)}|y|^{p} \leq G(y) \leq c_{5}\left[1+|y|^{p}\right] \text { for some } c_{5}>0 \text {, all } y \in \mathbb{R}^{N} \text {. }
$$

The examples that follow show that the framework provided by these conditions on $a(\cdot)$, is broad an includes as special cases many differential operators of interest (see Papageorgiou-Rădulescu [11]).

Example 1. (a) $a(y)=|y|^{p-2} y$ with $1<p<+\infty$.

This map corresponds to the $p$-Laplace differential operator defined by

$$
\Delta_{p} u=\operatorname{div}\left(|\nabla u|^{p-2} \nabla u\right) \quad \text { for all } u \in W^{1, p}(\Omega) .
$$

(b) $a(y)=|y|^{p-2} y+|y|^{q-2} y$ with $1<q<p<+\infty$.

This map corresponds to the $(p, q)$-Laplace differential operator defined by

$$
\Delta_{p} u+\Delta_{q} u \text { for all } u \in W^{1, p}(\Omega) .
$$

Such operators arise in many models of physical phenomena which involve the combination of two operators of different nature. We mention the works of Benci-D'Avenia-Fortunato-Pisani [2] (quantum physics - soliton solutions), Cherfils-Il'yasov [3] (reaction-diffusion systems) and Zhikov [21,22] (elasticity theory). A survey of some recent results concerning such equations, can be found in the paper of Marano-Mosconi [9]. Also, we mention the work of Papageorgiou-Vetro [17] on $(p, q)$-equations with variable exponents.

(c) $a(y)=\left[1+|y|^{2}\right]^{\frac{p-2}{2}} y \quad$ with $1<p<+\infty$.

This map corresponds to the modified capillary differential operator, given by

$$
\operatorname{div}\left[\left(1+|\nabla u|^{2}\right)^{\frac{p-2}{2}} \nabla u\right] \text { for all } u \in W^{1, p}(\Omega) .
$$

(d) $a(y)=|y|^{p-2} y\left[1+\frac{1}{1+|y|^{p}}\right] \quad$ with $1<p<+\infty$. 
The differential operator corresponding to this map is

$$
\Delta_{p} u+\operatorname{div}\left(\frac{|\nabla u|^{p-2}}{1+|\nabla u|^{2}} \nabla u\right) \quad \text { for all } u \in W^{1, p}(\Omega)
$$

and appears in problems of plasticity theory (see [11]).

By $A: W^{1, p}(\Omega) \rightarrow W^{1, p}(\Omega)^{*}$ we denote the nonlinear map defined by

$$
\langle A(u), h\rangle=\int_{\Omega}(a(\nabla u), \nabla h)_{\mathbb{R}^{N}} d z \quad \text { for all } u, h \in W^{1, p}(\Omega) .
$$

This map is bounded (that is, maps bounded sets to bounded sets), continuous and monotone (thus maximal monotone too). If $u, v \in W^{1, p}(\Omega)$ and $v \leq u$, then

$$
[v, u]=\left\{h \in W^{1, p}(\Omega): v(z) \leq h(z) \leq u(z) \text { for a.a. } z \in \Omega\right\} .
$$

Also by $\operatorname{int}_{C^{1}(\bar{\Omega})}[v, u]$ we denote the interior in the $C^{1}(\bar{\Omega})$-norm topology of $[v, u] \cap C^{1}(\bar{\Omega})$. Finally by $\delta_{k m}$ we denote the Kronecker symbol defined by

$$
\delta_{k m}=\left\{\begin{array}{ll}
1 & \text { if } k=m \\
0 & \text { if } k \neq m
\end{array}, \quad k, m \in \mathbb{N}_{0}\right.
$$

The hypotheses on the potential function $\xi(\cdot)$ and the boundary coefficient $\beta(\cdot)$ are:

$H(\xi): \xi(\cdot) \in L^{\infty}(\Omega), \xi(z) \geq 0$ for a.a. $z \in \Omega$.

$H(\beta): \beta(\cdot) \in C^{0, \alpha}(\partial \Omega)$ with $\alpha \in(0,1)$ and $\beta(z) \geq 0$ for all $z \in \partial \Omega$.

$H_{0}: \xi \not \equiv 0$ or $\beta \not \equiv 0$.

Remark 2. Evidently the case $\beta \equiv 0$ is included and corresponds to the Neumann problem.

The next lemma can be found in Mugnai-Papageorgiou [10] (Lemma 4.11). In the sequel by $\|\cdot\|$ we denote the norm of the Sobolev space $W^{1, p}(\Omega)$ defined by

$$
\|u\|=\left[\|u\|_{p}^{p}+\|\nabla u\|_{p}^{p}\right]^{1 / p} \quad \text { for all } u \in W^{1, p}(\Omega) .
$$

Lemma 2. If $\xi_{0}(\cdot) \in L^{\infty}(\Omega), \xi_{0}(z) \geq 0$ for a.a. $z \in \Omega$ and $\xi_{0} \not \equiv 0$, then there exists $c_{6}>0$ such that $\|\nabla u\|_{p}^{p}+\int_{\Omega} \xi_{0}(z)|u|^{p} d z \geq c_{6}\|u\|^{p}$ for all $W^{1, p}(\Omega)$.

Let $\sigma(\cdot)$ denote $(N-1)$-dimensional Hausdorff (surface) measure on $\partial \Omega$. Using this measure, we can define in the usual way the boundary Lebesgue spaces $L^{r}(\partial \Omega)$ with $1 \leq r \leq+\infty$. We know that there exists a unique continuous linear map $\gamma_{0}: W^{1, p}(\Omega) \rightarrow L^{p}(\partial \Omega)$, known as the "trace map", such that

$$
\gamma_{0}(u)=\left.u\right|_{\partial \Omega} \quad \text { for all } u \in W^{1, p}(\Omega) \cap C(\bar{\Omega}) .
$$


This map is compact, $\operatorname{im} \gamma_{0}=W^{\frac{1}{p^{\prime}}, p}(\partial \Omega)\left(\frac{1}{p}+\frac{1}{p^{\prime}}=1\right)$ and $\operatorname{ker} \gamma_{0}=$ $W_{0}^{1, p}(\Omega)$. In the sequel, for the sake of notational simplicity, we drop the use of the trace map $\gamma_{0}(\cdot)$. All restrictions of Sobolev functions are understood in the sense of traces.

The next lemma can be found in Gasiński-Papageorgiou [4] (Proposition $2.4)$.

Lemma 3. If $\beta_{0}(\cdot) \in L^{\infty}(\partial \Omega), \beta_{0}(z) \geq 0$ for $\sigma$-a.a. $z \in \partial \Omega$ and $\beta_{0} \not \equiv 0$, then $u \rightarrow\left[\|\nabla u\|_{p}^{p}+\int_{\partial \Omega} \beta_{0}(z)|u|^{p} d \sigma\right]^{1 / p}$ is an equivalent norm on $W^{1, p}(\Omega)$.

Consider the $C^{1}$-functional $\gamma: W^{1, p}(\Omega) \rightarrow \mathbb{R}$ defined by

$\gamma(u)=\int_{\Omega} p G(\nabla u) d z+\int_{\Omega} \xi(z)|u|^{p} d z+\int_{\partial \Omega} \beta(z)|u|^{p} d \sigma \quad$ for all $u \in W^{1, p}(\Omega)$.

From Corollary 1 and Lemmata 2 and 3, we infer that there exists $c_{7}>0$ such that

$$
c_{7}\|u\|^{p} \leq \gamma(u) \text { for all } u \in W^{1, p}(\Omega) .
$$

Now we introduce the conditions on the source term $f(z, x)$. Note that by $p^{*}>1$ we denote the critical Sobolev exponent corresponding to $p$ and defined by

$$
p^{*}= \begin{cases}\frac{N p}{N-p} & \text { if } N>p \\ +\infty & \text { if } p \geq N .\end{cases}
$$

$H(f): f: \Omega \times \mathbb{R} \rightarrow \mathbb{R}$ is a Carathéodory function such that $f(z, 0)=0$ for a.a. $z \in \Omega$ and

(i) there exist $\widehat{c}_{1}, \widehat{c}_{2}>0$ and $r \in\left(p, p^{*}\right)$ such that uniformly for a.a. $z \in \Omega$

$$
-\widehat{c}_{1} \leq \liminf _{x \rightarrow 0} \frac{f(z, x)}{|x|^{r-2} x} \leq \limsup _{x \rightarrow 0} \frac{f(z, x)}{|x|^{r-2} x} \leq \widehat{c}_{2}
$$

(ii) if $F(z, x)=\int_{0}^{x} f(z, s) d s$, then there exists $\tau \in\left(r, p^{*}\right)$ such that

$$
\lim _{x \rightarrow 0} \frac{F(z, x)}{|x|^{\tau}}=+\infty \quad \text { uniformly for a.a. } z \in \Omega
$$

(iii) if $x \neq 0$, then $f(z, x) \neq 0$ for all $z \in \Omega$.

Remark 3. We emphasize that conditions $(i)$ and $(i i)$ concern only the behavior of $f(z, \cdot)$ near zero. There are no conditions concerning the behavior of $x \rightarrow$ $f(z, x)$ for $|x|$ big. Also we point out that no local sign condition is assumed on $f(z, \cdot)$.

On account of hypotheses $H(f)(i),(i i)$ we see that we can find $\delta_{0} \in(0,1]$ and $c_{8}>1$ such that

$$
|f(z, x)| \leq c_{8}|x|^{r-1},|F(z, x)| \leq \frac{c_{8}}{r}|x|^{r}, F(z, x) \geq|x|^{\tau}
$$


for a.a. $z \in \Omega$, all $|x| \leq \delta_{0}$.

Let $\vartheta \in\left(0, \delta_{0}\right)$ and consider a cut-off function $\eta \in C_{c}^{1}(\mathbb{R})$ such that

$$
\operatorname{supp} \eta \subseteq[-\vartheta, \vartheta], \quad 0 \leq \eta \leq 1,\left.\quad \eta\right|_{[-\vartheta / 2, \vartheta / 2]} \equiv 1 .
$$

We introduce the following modification of the source term

$$
\widehat{f}(z, x)=\eta(x) f(z, x)+(1-\eta(x))|x|^{r-2} x .
$$

Evidently $\widehat{f}(z, x)$ is a Carathéodory function. We set $\widehat{F}(z, x)=\int_{0}^{x} \widehat{f}(z, s) d s$. Also, we consider the positive and negative truncations of $\widehat{f}(z, \cdot)$, namely the Carathéodory functions $\widehat{f}_{ \pm}(z, x)=\widehat{f}\left(z, \pm x^{ \pm}\right)$. We set $\widehat{F}_{ \pm}(z, x)=\int_{0}^{x} \widehat{f}_{ \pm}(z, s) d s$. From (2.3)-(2.5) it follows that

$$
\begin{aligned}
& |\widehat{f}(z, x)| \leq c_{9}|x|^{r-1} \quad \text { for a.a. } z \in \Omega, \text { all } x \in \mathbb{R}, \text { some } c_{9}>0, \\
\Rightarrow & |\widehat{F}(z, x)| \leq \frac{c_{9}}{r}|x|^{r} \quad \text { for a.a. } z \in \Omega, \text { all } x \in \mathbb{R} .
\end{aligned}
$$

Finally we recall the definition of critical groups for a $C^{1}$-functional at an isolated critical point. We will use critical groups to distinguish between critical points. So, let $X$ be a Banach space and $\varphi \in C^{1}(X)$. We introduce the following sets

$$
K_{\varphi}=\left\{u \in X: \varphi^{\prime}(u)=0\right\} \text { and } \varphi^{c}=\{u \in X: \varphi(u) \leq c\} \text { with } c \in \mathbb{R} .
$$

For every topological pair $\left(Y_{1}, Y_{2}\right)$ with $Y_{2} \subseteq Y_{1} \subseteq X$ and every integer $k \geq 0$, by $H_{k}\left(Y_{1}, Y_{2}\right)$ we denote the $k^{t h}$-singular homology group with integer coefficients. Then for $u \in K_{\varphi}$ isolated and $c=\varphi(u)$, the critical groups of $\varphi$ at $u$ are defined by

$$
C_{k}(\varphi, u)=H_{k}\left(\varphi^{c} \cap U, \varphi^{c} \cap U \backslash\{u\}\right) \text { for all } k \in \mathbb{N}_{0}
$$

with $U$ a neighborhood of $u$ such that $K_{\varphi} \cap \varphi^{c} \cap U=\{u\}$. The excision property of singular homology guarantees that this definition is independent of the choice of the isolating neighborhood.

\section{Constant sign solutions}

In this section we show that for all $\lambda>0$ big problem $\left(P_{\lambda}\right)$ has at least two smooth constant sign solutions.

Proposition 1. If hypotheses $H(f)$ hold, then the functions $\widehat{f}_{ \pm}(z, x)$ satisfy the AR-condition (the Ambrosetti-Rabinowitz condition, see [1]).

Proof. We do the proof for the positive truncation $\widehat{f}_{+}(z, x)$. The proof for the negative truncation is done similarly.

Let $x>\vartheta$. We have

$$
\begin{aligned}
& \widehat{F}(z, x)=\int_{0}^{x} \widehat{f}(z, s) d s=\int_{0}^{\vartheta}\left[\eta(s) f(z, s)+(1-\eta(s)) s^{r-1}\right] d s+\int_{\vartheta}^{x} s^{r-1} d s \\
& \leq \frac{c_{10}}{r} \vartheta^{r}+\frac{1}{r}\left[x^{r}-\vartheta^{r}\right] \quad \text { for some } c_{10}>0(\text { see }(2.3)-(2.5))
\end{aligned}
$$


Also since $x>\vartheta$, we have $\widehat{f}(z, x) x=|x|^{r}$ (see (2.4), (2.5)). Let $\mu \in(p, r)$. We have (see (2.6), (3.1)):

$$
\widehat{f}(z, x) x-\mu \widehat{F}(z, x) \geq\left[1-\frac{\mu}{r}\right] x^{r}-\frac{\mu}{r}\left[c_{8}-1\right] \vartheta^{r} .
$$

We choose $M_{+}>\vartheta$ big so that

$$
\left[1-\frac{\mu}{r}\right] M_{+}^{r} \geq \frac{\mu}{r}\left[c_{8}-1\right] \vartheta^{r}>0 \quad\left(\text { recall } c_{8}>1\right) .
$$

Using this in (3.2), we infer that

$$
\widehat{f}(z, x) x \geq \mu \widehat{F}(z, x) \quad \text { for a.a. } z \in \Omega, \text { all } x \geq M_{+}>\vartheta .
$$

Also note that

$$
\begin{aligned}
& \widehat{F}\left(z, M_{+}\right) \geq \int_{\vartheta}^{M_{+}} s^{r-1} d s=\frac{1}{r}\left[M_{+}^{r}-\vartheta^{r}\right] \quad \text { for a.a. } z \in \Omega, \\
\Rightarrow & \operatorname{essinf}_{\Omega} \widehat{F}\left(\cdot, M_{+}\right)>0 .
\end{aligned}
$$

Moreover, from the above it is also clear that

$$
\widehat{F}(z, x)>0 \text { for a.a. } z \in \Omega \text {, all } x \geq M_{+} \text {. }
$$

From (3.3)-(3.5) it follows that $\widehat{f}_{+}$satisfies the AR-condition. Similarly for $\widehat{f}_{-}(z, x)$.

We consider the following nonlinear parametric Robin problem

$$
\left\{\begin{array}{l}
-\operatorname{div} a(\nabla u(z))+\xi(z)|u(z)|^{p-2} u(z)=\lambda \widehat{f}(z, u(z)) \text { in } \Omega \\
\frac{\partial u}{\partial n_{a}}+\beta(z)|u|^{p-2} u=0 \text { on } \partial \Omega .
\end{array}\right.
$$

For this problem when $\lambda>0$ is big, we can have constant sign smooth solutions. Recall that the Banach space $C^{1}(\bar{\Omega})$ is ordered with positive (order) cone

$$
C_{+}=\left\{u \in C^{1}(\bar{\Omega}): u(z) \geq 0 \text { for all } z \in \bar{\Omega}\right\} .
$$

This cone has a nonempty interior given by

$$
\operatorname{int} C_{+}=\left\{u \in C_{+}: u(z)>0 \text { for all } z \in \bar{\Omega}\right\} \text {. }
$$

Proposition 2. If hypotheses $H(a), H(\xi), H(\beta), H_{0}, H(f)$ hold, then there exists $\lambda_{0}>0$ such that for all $\lambda \geq \lambda_{0}$ problem $\left(Q_{\lambda}\right)$ has a positive solution $u_{\lambda} \in \operatorname{int} C_{+}$and a negative solution $v_{\lambda} \in-\operatorname{int} C_{+}$.

Proof. We do the proof for the positive solution, the proof for the negative solution being similar.

We consider the $C^{1}$-functional $\widehat{\varphi}_{\lambda}^{+}: W^{1, p}(\Omega) \rightarrow \mathbb{R}$ defined by

$$
\widehat{\varphi}_{\lambda}^{+}(u)=\frac{1}{p} \gamma(u)-\lambda \int_{\Omega} \widehat{F}_{+}(z, u) d z=\frac{1}{p} \gamma(u)-\lambda \int_{\Omega} \widehat{F}\left(z, u^{+}\right) d z
$$


for all $u \in W^{1, p}(\Omega)$. Using (2.2) and (2.6), we have

$$
\begin{aligned}
\widehat{\varphi}_{\lambda}^{+}(u) & \geq c_{7}\|u\|^{p}-\lambda c_{11}\|u\|^{r} \quad \text { for some } c_{11}>0, \text { all } u \in W^{1, p}(\Omega) \\
& =\left[c_{7}-\lambda c_{11}\|u\|^{r-p}\right]\|u\|^{p} .
\end{aligned}
$$

We see that, if we choose $\rho_{\lambda}<\left[c_{7} /\left(\lambda c_{11}\right)\right]^{\frac{1}{r-p}}$, then

$$
\widehat{\varphi}_{\lambda}^{+}(u) \geq \widehat{m}_{\lambda}^{+}>0 \quad \text { for all } u \in W^{1, p}(\Omega) \text { with }\|u\|=\rho_{\lambda} .
$$

Let $\bar{u}_{+}=\vartheta / 2 \in W^{1, p}(\Omega)$. We have (see $\left.(2.3)-(2.5)\right)$ :

$$
\begin{aligned}
\widehat{\varphi}_{\lambda}^{+}\left(\bar{u}_{+}\right) \leq \frac{\vartheta^{p}}{p}\left[\|\xi\|_{1}+\|\beta\|_{L^{1}(\partial \Omega)}\right]-\lambda \vartheta^{\tau}|\Omega|_{N} \\
\Rightarrow \widehat{\varphi}_{\lambda}^{+}\left(\bar{u}_{+}\right) \leq c_{12}-\lambda c_{13} \quad \text { for some } c_{12}, c_{13}>0 .
\end{aligned}
$$

Here by $|\Omega|_{N}$ we denote the Lebesgue measure of $\Omega$.

Note that $\rho_{\lambda} \rightarrow 0^{+}$as $\lambda \rightarrow+\infty$. So, we can find $\lambda_{0}^{+} \geq \frac{c_{12}}{c_{13}}$ such that

$$
\widehat{\varphi}_{\lambda}^{+}\left(\bar{u}_{+}\right) \leq 0 \text { and }\left\|\bar{u}_{+}\right\|>\rho_{\lambda} \quad \text { for all } \lambda \geq \lambda_{0}^{+} \text {. }
$$

From Proposition 1 we know that $\widehat{f}_{+}$satisfies the AR-condition. It follows that

$$
\widehat{\varphi}_{\lambda}^{+} \text {satisfies the PS-condition. }
$$

From (3.6)-(3.8) we see that we can apply the mountain pass theorem and find $u_{\lambda} \in W^{1, p}(\Omega)$ such that

$$
u_{\lambda} \in K_{\widehat{\varphi}_{\lambda}^{+}} \text {and } \widehat{\varphi}_{\lambda}^{+}(0)=0<\widehat{m}_{\lambda}^{+} \leq \widehat{\varphi}_{\lambda}^{+}\left(u_{\lambda}\right) .
$$

From (3.9) we have $u_{\lambda} \neq 0$ and

$$
\begin{gathered}
\left(\widehat{\varphi}_{\lambda}^{+}\right)^{\prime}\left(u_{\lambda}\right)=0, \quad \Rightarrow \quad\left\langle A\left(u_{\lambda}\right), h\right\rangle+\int_{\Omega} \xi(z)\left|u_{\lambda}\right|^{p-2} u_{\lambda} h d z \\
+\int_{\partial \Omega} \beta(z)\left|u_{\lambda}\right|^{p-2} u_{\lambda} h d \sigma=\lambda \int_{\Omega} \widehat{f}\left(z, u_{\lambda}^{+}\right) h d z
\end{gathered}
$$

for all $h \in W^{1, p}(\Omega)$. In (3.10) we choose $h=-u_{\lambda}^{-} \in W^{1, p}(\Omega)$ and obtain

$$
\left.\frac{c_{1}}{p-1}\left\|\nabla u_{\lambda}^{-}\right\|_{p}^{p}+\int_{\Omega} \xi(z)\left(u_{\lambda}^{-}\right)^{p} d z+\int_{\partial \Omega} \beta(z)\left(u_{\lambda}^{-}\right)^{p} d \sigma \leq 0 \text { (see Lemma } 1\right)
$$

$\Rightarrow c_{14}\left\|u_{\lambda}^{-}\right\|^{p} \leq 0$ for some $c_{14}>0$ (see Lemmata 2, 3 and hypothesis $H_{0}$ ),

$\Rightarrow u_{\lambda} \geq 0, u_{\lambda} \neq 0$.

From (3.10) we have

$$
\left\{\begin{array}{l}
-\operatorname{div} a\left(\nabla u_{\lambda}\right)+\xi(z) u_{\lambda}^{p-1}=\lambda f\left(z, u_{\lambda}\right) \text { for a.a. } z \in \Omega \\
\quad \frac{\partial u_{\lambda}}{\partial n_{a}}+\beta(z) u_{\lambda}^{p-1}=0 \text { on } \partial \Omega
\end{array}\right.
$$


From (3.11) and Proposition 2.10 of Papageorgiou-Rădulescu [12], we have that $u_{\lambda} \in L^{\infty}(\Omega)$. Hence from Lieberman [8], we infer that $u_{\lambda} \in C_{+} \backslash\{0\}$. From (3.11) we have

$$
\operatorname{div} a\left(\nabla u_{\lambda}\right) \leq\left[\|\xi\|_{\infty}+\lambda\left\|u_{\lambda}\right\|_{\infty}^{r-p}\right] u_{\lambda}^{p-1} \quad \text { for a.a. } z \in \Omega, \Rightarrow u_{\lambda} \in \operatorname{int} C_{+}
$$

(from the nonlinear maximum principle, see Pucci-Serrin [19]).

Similarly, if we work with the $C^{1}$-functional $\widehat{\varphi}_{\lambda}^{-}: W^{1, p}(\Omega) \rightarrow \mathbb{R}$ defined by

$$
\widehat{\varphi}_{\lambda}^{-}(u)=\frac{1}{p} \gamma(u)-\lambda \int_{\Omega} \widehat{F}_{-}(z, u) d z=\frac{1}{p} \gamma(u)-\lambda \int_{\Omega} \widehat{F}\left(z,-u^{-}\right) d z
$$

for all $u \in W^{1, p}(\Omega)$, via the mountain pass theorem, we produce a negative solution $v_{\lambda} \in-\operatorname{int} C_{+}$for all $\lambda \geq \lambda_{0}^{-}$.

Let $\lambda_{0}=\max \left\{\lambda_{0}^{+}, \lambda_{0}^{-}\right\}$. Then for $\lambda \geq \lambda_{0}$ problem $\left(Q_{\lambda}\right)$ has two constant sign solutions $u_{\lambda} \in \operatorname{int} C_{+}, v_{\lambda} \in-\operatorname{int} C_{+}$.

Proposition 3. If hypotheses $H(a), H(\xi), H(\beta), H_{0}, H(f)$ hold and $\lambda \geq \lambda_{0}$, then $u_{\lambda}, v_{\lambda} \rightarrow 0$ in $C^{1}(\bar{\Omega})$ as $\lambda \rightarrow+\infty$.

Proof. From the proof of Proposition 2 we know that

$$
\widehat{\varphi}_{\lambda}^{+}\left(u_{\lambda}\right)=\inf _{\widehat{\gamma} \in \Gamma} \max _{0 \leq t \leq 1} \widehat{\varphi}_{\lambda}^{+}(\widehat{\gamma}(t)),
$$

where $\Gamma=\left\{\widehat{\gamma} \in C\left([0,1], W^{1, p}(\Omega)\right): \widehat{\gamma}(0)=0, \widehat{\gamma}(1)=\bar{u}_{+}\right\}$(since $u_{\lambda} \in K_{\varphi_{\lambda}^{+}}$is of mountain pass type). From (2.3) and (2.5) we see that

$$
\widehat{F}(z, x) \geq c_{15}|x|^{\tau} \text { for a.a. } z \in \Omega \text {, all }|x| \leq \delta_{0} \text {, some } c_{15}>0 .
$$

Consider the function $k_{\lambda}: \mathbb{R}_{+} \rightarrow \mathbb{R}$ defined by

$$
k_{\lambda}(t)=\frac{t^{p} \bar{u}_{+}^{p}}{p}\left[\|\xi\|_{1}+\|\beta\|_{L^{1}(\partial \Omega)}\right]-\lambda c_{15} t^{\tau} \bar{u}_{+}^{\tau}|\Omega|_{N}, \quad t>0 .
$$

This function is differentiable, unbounded below (recall that $\tau>p$ ) and attains its supremum on $\mathbb{R}_{+}$at $t_{0}>0$. Let $c_{16}=\frac{\bar{u}_{+}^{p}}{p}\left[\|\xi\|_{1}+\|\beta\|_{L^{1}(\partial \Omega)}\right]>0$ and $c_{17}=c_{15} \bar{u}_{+}^{\tau}|\Omega|_{N}>0$. We have

$$
k_{\lambda}^{\prime}\left(t_{0}\right)=0, \quad \Rightarrow \quad p c_{16} t_{0}^{p-1}=\lambda \tau c_{17} t_{0}^{\tau-1}, \quad \Rightarrow \quad t_{0}=\left[\frac{p c_{16}}{\lambda \tau c_{17}}\right]^{\frac{1}{\tau-p}} .
$$

We have

$$
k_{\lambda}\left(t_{0}\right) \leq c_{18} / \lambda^{\frac{p}{\tau-p}} \quad \text { for some } c_{18}>0, \text { all } \lambda \geq \lambda_{0} .
$$

On account of (3.12) we have

$$
\begin{gathered}
\widehat{\varphi}_{\lambda}^{+}\left(u_{\lambda}\right) \leq \max _{0 \leq t \leq 1} \widehat{\varphi}_{\lambda}^{+}\left(t \bar{u}_{+}\right) \leq \max _{0 \leq t \leq 1} k_{\lambda}(t) \leq k_{\lambda}\left(t_{0}\right) \quad\left(\text { since } t_{0}>0\right. \text { is } \\
\text { the maximizer of } \left.k_{\lambda}(\cdot) \text { on } \mathbb{R}_{+}\right) \leq c_{18} / \lambda^{\frac{p}{\tau-p}} .
\end{gathered}
$$


Now consider a sequence $\lambda_{n} \rightarrow+\infty$ and let $u_{n}=u_{\lambda_{n}} \in \operatorname{int} C_{+}$for all $n \in \mathbb{N}$. With $\mu \in(p, r)$ as in the proof of Proposition 1 , we have

$$
\mu \widehat{\varphi}_{\lambda}^{+}\left(u_{n}\right)=\frac{\mu}{p} \gamma\left(u_{n}\right)-\lambda_{n} \int_{\Omega} \mu \widehat{F}\left(z, u_{n}\right) d z \leq \mu c_{18} / \lambda_{n}^{\frac{p}{\tau-p}}
$$

for all $n \in \mathbb{N}$ (see (3.14)). Also, for all $n \in \mathbb{N}$, we have

$$
\begin{aligned}
& \left\langle\left(\widehat{\varphi}_{\lambda_{n}}^{+}\right)^{\prime}\left(u_{n}\right), u_{n}\right\rangle=0 \Rightarrow-\int_{\Omega}\left(a\left(\nabla u_{n}\right), \nabla u_{n}\right)_{\mathbb{R}^{N}} d z \\
& \quad-\int_{\Omega} \xi(z) u_{n}^{p} d z-\int_{\partial \Omega} \beta(z) u_{n}^{p} d \sigma+\lambda_{n} \int_{\Omega} \widehat{f}\left(z, u_{n}\right) u_{n} d z=0 .
\end{aligned}
$$

Adding (3.15) and (3.16), we obtain

$$
\begin{aligned}
& (\mu-p) \int_{\Omega} G\left(\nabla u_{n}\right) d z+\int_{\Omega}\left[p G\left(\nabla u_{n}\right)-\left(a\left(\nabla u_{n}\right), \nabla u_{n}\right)_{\mathbb{R}^{N}}\right] d z \\
& +\left(\frac{\mu}{p}-1\right) \int_{\Omega} \xi(z) u_{n}^{p} d z+\left(\frac{\mu}{p}-1\right) \int_{\partial \Omega} \beta(z) u_{n}^{p} d \sigma \\
& +\lambda_{n} \int_{\Omega}\left[\widehat{f}\left(z, u_{n}\right) u_{n}-\mu \widehat{F}\left(z, u_{n}\right)\right] d z \leq \mu c_{18} / \lambda_{n}^{\frac{p}{\tau-p}} \leq \mu c_{18} / \lambda_{1}^{\frac{p}{\tau-p}}
\end{aligned}
$$

Using Lemma 1, hypothesis $H(a)(i v)$ and Proposition 1 (see its proof), we obtain

$$
\begin{aligned}
& \left(\frac{\mu}{p}-1\right) c_{1}\left\|\nabla u_{n}\right\|_{p}^{p}+\left(\frac{\mu}{p}-1\right) \int_{\Omega} \xi(z) u_{n}^{p} d z+\left(\frac{\mu}{p}-1\right) \int_{\partial \Omega} \beta(z) u_{n}^{p} d \sigma \\
& \leq c_{19} / \lambda_{1}^{\frac{p}{\tau-p}} \text { for some } c_{19}>0 \text {, all } n \in \mathbb{N}, \Rightarrow\left\{u_{n}\right\}_{n \geq 1} \subseteq W^{1, p}(\Omega) \text { is bounded }
\end{aligned}
$$

(see Lemmata 2, 3 and recall $\mu>p$ ). Recall that for all $n \in \mathbb{N}$, we have

$$
\left\{\begin{array}{l}
-\operatorname{div} a\left(\nabla u_{n}\right)+\xi(z) u_{n}^{p-1}=\lambda_{n} \widehat{f}\left(z, u_{n}\right) \text { for a.a. } z \in \Omega \\
\quad \frac{\partial u_{n}}{\partial n_{a}}+\beta(z) u_{n}^{p-1}=0 \text { on } \partial \Omega .
\end{array}\right.
$$

Then from (3.17), (3.18) and Proposition 2.10 of Papageorgiou-Rădulescu [12], we know that we can find $c_{20}>0$ such that

$$
u_{n} \in L^{\infty}(\Omega) \text { and }\left\|u_{n}\right\|_{\infty} \leq c_{20} \text { for all } n \in \mathbb{N} .
$$

Then the nonlinear regularity theory of Lieberman [8] implies that there exist $\alpha \in(0,1)$ and $c_{21}>0$ such that

$$
u_{n} \in C^{1, \alpha}(\bar{\Omega}) \text { and }\left\|u_{n}\right\|_{C^{1, \alpha}(\bar{\Omega})} \leq c_{21} \quad \text { for all } n \in \mathbb{N} .
$$

On account of (3.19) and the compact embedding of $C^{1, \alpha}(\bar{\Omega})$ into $C^{1}(\bar{\Omega})$, we have, at least for a subsequence, that $u_{n} \rightarrow \widehat{u}$ in $C^{1}(\bar{\Omega})$. We must have $\widehat{u} \equiv 0$ or otherwise from (3.18) and hypothesis $H(f)(i i i)$, we have a contradiction. 
Therefore we conclude that $\lambda \rightarrow+\infty \Rightarrow u_{\lambda} \rightarrow 0$ in $C^{1}(\bar{\Omega})$. Similarly we show that $\lambda \rightarrow+\infty \Rightarrow v_{\lambda} \rightarrow 0$ in $C^{1}(\bar{\Omega})$.

We introduce the following two solution sets: $\widehat{S}_{\lambda}^{+}=\{$set of positive solutions of $\left.\left(Q_{\lambda}\right)\right\}, \widehat{S}_{\lambda}^{-}=\left\{\right.$set of negative solutions of $\left.\left(Q_{\lambda}\right)\right\}$.

From Proposition 2, we have

$$
\emptyset \neq \widehat{S}_{\lambda}^{+} \subseteq \operatorname{int} C_{+} \text {and } \emptyset \neq \widehat{S}_{\lambda}^{-} \subseteq-\operatorname{int} C_{+} \text {for all } \lambda \geq \lambda_{0} .
$$

Moreover, from Papageorgiou-Rădulescu-Repovš [13] (see the proof of Proposition 3.2), we have that

$\widehat{S}_{\lambda}^{+}$is downward directed

(that is, if $u_{1}, u_{2} \in \widehat{S}_{\lambda}^{+}$, then we can find $\widehat{u} \in \widehat{S}_{\lambda}^{+}$such that $\widehat{u} \leq u_{1}, \widehat{u} \leq u_{2}$ ), $\widehat{S}_{\lambda}^{-}$is upward directed

(that is, if $v_{1}, v_{2} \in \widehat{S}_{\lambda}^{-}$, then we can find $\widehat{v} \in \widehat{S}_{\lambda}^{-}$such that $v_{1} \leq \widehat{v}, v_{2} \leq \widehat{v}$ ).

Proposition 4. If hypotheses $H(a), H(\xi), H(\beta), H_{0}, H(f)$ hold and $\lambda \geq \lambda_{0}$, then problem $\left(Q_{\lambda}\right)$ has a smallest positive solution $u_{\lambda}^{*} \in \widehat{S}_{\lambda}^{+} \subseteq \operatorname{int} C_{+}$and a biggest negative solution $v_{\lambda}^{*} \in \widehat{S}_{\lambda}^{-} \subseteq-\operatorname{int} C_{+}$.

Proof. Since $\widehat{S}_{\lambda}^{+}$is downward directed, using Lemma 3.10, p. 178, of HuPapageorgiou [6], we can find a decreasing sequence $\left\{u_{n}\right\}_{n \geq 1} \subseteq \widehat{S}_{\lambda}^{+}$such that

$$
\inf _{n \geq 1} u_{n}=\inf \widehat{S}_{\lambda}^{+} \text {and } 0 \leq u_{n} \leq u_{1} \quad \text { for all } n \in \mathbb{N} \text {. }
$$

We have

$$
\left\langle A\left(u_{n}\right), h\right\rangle+\int_{\Omega} \xi(z) u_{n}^{p-1} h d z+\int_{\partial \Omega} \beta(z) u_{n}^{p-1} h d \sigma=\lambda \int_{\Omega} \widehat{f}\left(z, u_{n}\right) h d z
$$

for all $h \in W^{1, p}(\Omega)$, all $n \in \mathbb{N}$. Choosing $h=u_{n} \in W^{1, p}(\Omega)$ in (3.21) and using (3.20), we infer that

$$
\left\{u_{n}\right\}_{n \geq 1} \subseteq W^{1, p}(\Omega) \text { is bounded. }
$$

Then as before (see the proof of Proposition 3), using the nonlinear regularity theory, we show that

$$
u_{n} \rightarrow u_{\lambda}^{*} \text { in } C^{1}(\bar{\Omega}) \text { as } n \rightarrow+\infty .
$$

Using $h=u_{n} \in W^{1, p}(\Omega)$ in (3.21) and on account of Lemma 1, we have

$$
\begin{aligned}
& \frac{c_{1}}{p-1}\left\|\nabla u_{n}\right\|_{p}^{p}+\int_{\Omega} \xi(z)\left|u_{n}\right|^{p} d z+\int_{\partial \Omega} \beta(z)\left|u_{n}\right|^{p} d \sigma \\
& \leq \lambda \int_{\Omega} \widehat{f}\left(z, u_{n}\right) u_{n} d z \quad \text { for all } n \in \mathbb{N}, \\
\Rightarrow \quad & c_{22}\left\|u_{n}\right\|^{p} \leq \lambda \int_{\Omega} \widehat{f}\left(z, u_{n}\right) u_{n} d z \quad \text { for some } c_{22}>0, \text { all } n \in \mathbb{N}
\end{aligned}
$$

(see Lemmata 2 and 3). 
Suppose that $u_{\lambda}^{*}=0$. Then

$$
u_{n} \rightarrow 0 \text { in } C^{1}(\bar{\Omega})(\operatorname{see}(3.22)) .
$$

Let $y_{n}=u_{n} /\left\|u_{n}\right\|, n \in \mathbb{N}$. Then $\left\|y_{n}\right\|=1$ for all $n \in \mathbb{N}$ and so we may assume that

$$
y_{n} \stackrel{w}{\rightarrow} y \text { in } W^{1, p}(\Omega) \text { and } y_{n} \rightarrow y \text { in } L^{p}(\Omega) \text { and in } L^{p}(\partial \Omega) .
$$

On account of $(2.5),(3.24)$ and hypothesis $H(f)(i)$, we have

$$
\frac{f\left(z, u_{n}\right)}{u_{n}^{p-1}}=\frac{f\left(z, u_{n}\right)}{u_{n}^{r-1}} u_{n}^{r-p} \rightarrow 0 \quad \text { for a.a. } z \in \Omega .
$$

From (3.23) and via Fatou's lemma, we have

$$
0<c_{22} \leq \limsup _{n \rightarrow+\infty} \int_{\Omega} \frac{\widehat{f}\left(z, u_{n}\right)}{u_{n}^{r-1}} u_{n}^{r-p} y_{n} d z \leq 0,
$$

a contradiction. So, $u_{\lambda}^{*} \neq 0$. Passing to the limit as $n \rightarrow+\infty$ in (3.21) we conclude that

$$
u_{\lambda}^{*} \in \widehat{S}_{\lambda}^{+} \text {and } u_{\lambda}^{*}=\inf \widehat{S}_{\lambda}^{+} .
$$

Similarly we obtain

$$
v_{\lambda}^{*} \in \widehat{S}_{\lambda}^{-} \text {and } v_{\lambda}^{*}=\sup \widehat{S}_{\lambda}^{-} .
$$

Note that in this case, since $\widehat{S}_{\lambda}^{-}$is upward directed, we can find an increasing sequence $\left\{v_{n}\right\}_{n \geq 1} \subseteq \widehat{S}_{\lambda}^{-}$such that $\sup _{n \geq 1} v_{n}=\sup \widehat{S}_{\lambda}^{-}$.

From Proposition 3, we have at once the following corollary.

Corollary 2. If hypotheses $H(a), H(\xi), H(\beta), H_{0}, H(f)$ hold and $\lambda \geq \lambda_{0}$, then $u_{\lambda}^{*}, v_{\lambda}^{*} \rightarrow 0$ in $C^{1}(\bar{\Omega})$ as $\lambda \rightarrow+\infty$.

\section{Nodal solutions}

In this section, using the extremal constant sign solutions produced in Proposition 4 , we will produce a nodal solution.

Proposition 5. If hypotheses $H(a), H(\xi), H(\beta), H_{0}, H(f)$ hold, then there exists $\lambda^{*} \geq \lambda_{0}$ such that for all $\lambda>\lambda^{*}$ problem $\left(Q_{\lambda}\right)$ has a nodal (sign changing) solution.

Proof. Let $u_{\lambda}^{*} \in \operatorname{int} C_{+}$and $v_{\lambda}^{*} \in-\operatorname{int} C_{+}$be the two extremal constant sign solutions of problem $\left(Q_{\lambda}\right)$ produced in Proposition 4. We introduce the following truncation of the source term of the problem

$$
k(z, x)= \begin{cases}\widehat{f}\left(z, v_{\lambda}^{*}(z)\right) & \text { if } x<v_{\lambda}^{*}(z) \\ \widehat{f}(z, x) & \text { if } v_{\lambda}^{*}(z) \leq x \leq u_{\lambda}^{*}(z), \\ \widehat{f}\left(z, u_{\lambda}^{*}(z)\right) & \text { if } u_{\lambda}^{*}(z)<x\end{cases}
$$


This is a Carathéodory function. We also consider the positive and negative truncations of $k(z, \cdot)$, namely the Carathéodory functions $k_{ \pm}(z, x)=k\left(z, \pm x^{ \pm}\right)$ (recall $\left.x^{ \pm}=\max \{ \pm x, 0\}\right)$. We set

$$
K(z, x)=\int_{0}^{x} k(z, s) d s \text { and } K_{ \pm}(z, x)=\int_{0}^{x} k_{ \pm}(z, s) d s .
$$

We consider the $C^{1}$-functionals $\psi_{\lambda}, \psi_{\lambda}^{ \pm}: W^{1, p}(\Omega) \rightarrow \mathbb{R}$ defined by

$$
\begin{aligned}
\psi_{\lambda}(u) & =\frac{1}{p} \gamma(u)-\lambda \int_{\Omega} K(z, u) d z \\
\psi_{\lambda}^{ \pm}(u) & =\frac{1}{p} \gamma(u)-\lambda \int_{\Omega} K_{ \pm}(z, u) d z \quad \text { for all } u \in W^{1, p}(\Omega) .
\end{aligned}
$$

Using (4.1) we can easily see that

$$
K_{\psi_{\lambda}} \subseteq\left[v_{\lambda}^{*}, u_{\lambda}^{*}\right] \cap C^{1}(\bar{\Omega}), \quad K_{\psi_{\lambda}^{+}} \subseteq\left[0, u_{\lambda}^{*}\right] \cap C_{+}, \quad K_{\psi_{\lambda}^{-}} \subseteq\left[v_{\lambda}^{*}, 0\right] \cap\left(-C_{+}\right) .
$$

The extremality of $u_{\lambda}^{*} \in \operatorname{int} C_{+}$and of $v_{\lambda}^{*} \in-\operatorname{int} C_{+}$implies that

$$
K_{\psi_{\lambda}} \subseteq\left[v_{\lambda}^{*}, u_{\lambda}^{*}\right] \cap C^{1}(\bar{\Omega}), \quad K_{\psi_{\lambda}^{+}}=\left\{0, u_{\lambda}^{*}\right\}, \quad K_{\psi_{\lambda}^{-}}=\left\{0, v_{\lambda}^{*}\right\} .
$$

From $(4.1),(2.4)$ and Lemmata 2 and 3, it follows that $\psi_{\lambda}^{+}$is coercive.

Using the Sobolev embedding theorem, we see that $\psi_{\lambda}^{+}$is sequentially weakly lower semicontinuous. So, by the Weierstrass-Tonelli theorem, we can find $\widetilde{u}_{\lambda}^{*} \in W^{1, p}(\Omega)$ such that

$$
\psi_{\lambda}^{+}\left(\widetilde{u}_{\lambda}^{*}\right)=\inf \left[\psi_{\lambda}^{+}(u): u \in W^{1, p}(\Omega)\right] .
$$

Hypothesis $H(a)(i v)$ and Corollary 1 imply that there exists $c_{23}>0$ such that

$$
G(y) \leq c_{23}\left[|y|^{q}+|y|^{p}\right] \quad \text { for all } y \in \mathbb{R}^{N} .
$$

Let $\widehat{u}_{1}(q)$ denote the positive principal eigenfunction of the $q$-Laplacian with Robin boundary condition. We know that $\widehat{u}_{1}(q) \in \operatorname{int} C_{+}$(see [11]). Also $\operatorname{int}_{C^{1}(\bar{\Omega})}\left[\frac{1}{2} u_{\lambda}^{*}, u_{\lambda}^{*}\right] \neq \emptyset$. Then on account of Proposition 4.1.22, p. 274, of Papageorgiou-Rădulescu-Repovš [16], we can find $\widehat{t}>0$ such that

$$
\widehat{t} \widehat{u}_{1}(q) \in\left[u_{\lambda}^{*} / 2, u_{\lambda}^{*}\right] \cap \operatorname{int} C_{+} .
$$

From Corollary 2, we see that there exists $\lambda_{0}^{*} \geq \lambda_{0}$ such that

$$
\begin{aligned}
& u_{\lambda}^{*}(z) \in\left(0, \delta_{0}\right] \quad \text { for all } z \in \bar{\Omega}, \text { all } \lambda \geq \lambda_{0}^{*}, \\
\Rightarrow \quad & \widehat{F}\left(z, \widehat{t} \widehat{u}_{1}(q)(z)\right) \geq \widehat{t}^{\tau} \widehat{u}_{1}(q)(z) \quad \text { for a.a. } z \in \Omega \\
& \left(\text { see }(2.3),(2.5) \text { and recall } \delta_{0} \leq 1, \tau>r\right) .
\end{aligned}
$$

So, we have

$$
\psi_{\lambda}^{+}\left(\widehat{t} \widehat{u}_{1}(q)\right) \leq c_{23}\left[\widehat{t}^{q} \widehat{\lambda}_{1}(q)+\widehat{t}^{p}\left\|\nabla \widehat{u}_{1}(q)\right\|_{p}^{p}\right]-\lambda \widehat{t}^{\tau}\left\|\widehat{u}_{1}(q)\right\|_{\tau}^{\tau}
$$


(see (4.4), (4.1), (4.5), (4.6)).

Then, if $\lambda_{1}^{*}=c_{23}\left[\widehat{t}^{q} \widehat{\lambda}_{1}(q)+\widehat{t}^{p}\left\|\nabla \widehat{u}_{1}(q)\right\|_{p}^{p}\right] / \widehat{t}^{\tau}\left\|\widehat{u}_{1}(q)\right\|_{\tau}^{\tau}$, for $\lambda>\lambda^{*}$ $=\max \left\{\lambda_{0}^{*}, \lambda_{1}^{*}\right\}$ we have

$$
\begin{aligned}
& \psi_{\lambda}^{+}\left(\widehat{t}_{u_{1}}(q)\right)<0, \quad \Rightarrow \quad \psi_{\lambda}^{+}\left(\widetilde{u}_{\lambda}^{*}\right)<0=\psi_{\lambda}^{+}(0) \quad(\text { see }(4.3)), \\
\Rightarrow \quad & \widetilde{u}_{\lambda}^{*} \neq 0 \text { and } \widetilde{u}_{\lambda}^{*} \in K_{\psi_{\lambda}^{+}}, \Rightarrow \quad \widetilde{u}_{\lambda}^{*}=u_{\lambda}^{*} \in \operatorname{int} C_{+} .
\end{aligned}
$$

From (4.1) it is clear that $\left.\psi_{\lambda}\right|_{C_{+}}=\left.\psi_{\lambda}^{+}\right|_{C_{+}}$. Then from (4.3) and (4.7) it follows that

$$
\begin{aligned}
& u_{\lambda}^{*} \text { is a local } C^{1}(\bar{\Omega}) \text {-minimizer of } \psi_{\lambda}, \\
\Rightarrow \quad & u_{\lambda}^{*} \text { is a local } W^{1, p}(\Omega) \text {-minimizer of } \psi_{\lambda}, \lambda>\lambda^{*}, \\
& \text { (see Papageorgiou-Rădulescu [12], Proposition 2.12). }
\end{aligned}
$$

Similarly using this time the functional $\psi_{\lambda}^{-}$, we show that $v_{\lambda}^{*}$ is a local $W^{1, p}(\Omega)$-minimizer of $\psi_{\lambda}, \lambda>\lambda^{*}$. The functional $\psi_{\lambda}$ is coercive and so it satisfies the PS-condition (see Papageorgiou-Rădulescu-Repovš [16], Proposition 5.1.15). Since we have two distinct local minimizers, we can apply the mountain pass theorem and obtain $y_{\lambda} \in W^{1, p}(\Omega)$ such that

$$
y_{\lambda} \in K_{\psi_{\lambda}} \subseteq\left[v_{\lambda}^{*}, u_{\lambda}^{*}\right] \cap C^{1}(\bar{\Omega})(\text { see }(4.2)) \text { and } y_{\lambda} \notin\left\{u_{\lambda}^{*}, v_{\lambda}^{*}\right\} \text {. }
$$

From Theorem 6.5.8 of Papageorgiou-Rădulescu-Repovš [16], we have

$$
C_{1}\left(\psi_{\lambda}, y_{\lambda}\right) \neq 0
$$

On the other hand from the proof of Proposition 2, we know that for $\lambda>\lambda^{*}$, $u=0$ is a local minimizer of $\psi_{\lambda}$. Hence

$$
C_{k}\left(\psi_{\lambda}, 0\right)=\delta_{k 0} \mathbb{Z} \quad \text { for all } k \in \mathbb{N}_{0}
$$

From (4.8)-(4.10), it follows that $y_{\lambda} \in C^{1}(\bar{\Omega})$ is a nontrivial solution of $\left(Q_{\lambda}\right) \lambda>\lambda^{*}$, distinct from $\left\{u_{\lambda}^{*}, v_{\lambda}^{*}\right\}$. Since $y_{\lambda} \in\left[v_{\lambda}^{*}, u_{\lambda}^{*}\right]$, the extremality of $u_{\lambda}^{*}$ and $v_{\lambda}^{*}$ implies that $y_{\lambda} \in C^{1}(\bar{\Omega})$ is nodal.

Finally using Corollary 2 and (2.5), we have the following multiplicity result for problem $\left(P_{\lambda}\right)$.

Theorem 1. If hypotheses $H(a), H(\xi), H(\beta), H_{0}, H(f)$ hold, then there exists $\widehat{\lambda}^{*} \geq \lambda^{*}$ such that for all $\lambda>\widehat{\lambda}^{*}$ problem $\left(P_{\lambda}\right)$ has at least three nontrivial smooth solutions $u_{\lambda} \in \operatorname{int} C_{+}, v_{\lambda} \in-\operatorname{int} C_{+}, y_{\lambda} \in\left[v_{\lambda}, u_{\lambda}\right] \cap C^{1}(\bar{\Omega})$ nodal.

\section{References}

[1] A. Ambrosetti and P.H. Rabinowitz. Dual variational methods in critical point theory and applications. J. Functional Anal., 14(4):349-381, 1973. https://doi.org/10.1016/0022-1236(73)90051-7. 
[2] V. Benci, P. D'Avenia, D. Fortunato and L. Pisani. Solitons in several space dimensions: Derrick's problem and infinitely many solutions. Arch. Ration. Mech. Anal., 154:297-324, 2000. https://doi.org/10.1007/s002050000101.

[3] L. Cherfils and Y. Il'yasov. On the stationary solutions of generalized reaction diffusion equations with $p \& q$-Laplacian. Commun. Pure Appl. Anal., 4(1):9-22, 2005. https://doi.org/10.3934/cpaa.2005.4.9.

[4] L. Gasiński and N.S. Papageorgiou. Positive solutions for the Robin $p$-Laplacian problem with competing nonlinearities. Adv. Calc. Var., 12(1):31-58, 2019. https://doi.org/10.1515/acv-2016-0039.

[5] U. Guarnotta, S.A. Marano and N.S. Papageorgiou. Multiple nodal solutions to a Robin problem with sign-changing potential and locally defined reaction. Rend. Lincei Mat. Appl., 30(2):269-294, 2019. https://doi.org/10.4171/RLM/847.

[6] S. Hu and N.S. Papageorgiou. Handbook of Multivalued Analysis. Vol. I. Theory. Mathematics and its Applications 419. Kluwer Academic Publishers, Dordrecht, The Netherlands, 1997. https://doi.org/10.1007/978-1-4615-4665-8_17.

[7] Z. Li and Z.-Q. Wang. Schrödinger equations with concave and convex nonlinearities. Z. Angew. Math. Phys., 56:609-629, 2005. https://doi.org/10.1007/s00033005-3115-6.

[8] G.M. Lieberman. The natural generalization of the natural conditions of Ladyzhenskaya and Ural'tseva for elliptic equations. Comm. Partial Diff. Equations, 16(2-3):311-361, 1991. https://doi.org/10.1080/03605309108820761.

[9] S.A. Marano and S. Mosconi. Some recent results on the Dirichlet problem for $(p, q)$-Laplace equations. Discr. Cont. Dyn. Systems, Ser. S, 11(2):279-291, 2018. https://doi.org/10.3934/dcdss.2018015.

[10] D. Mugnai and N.S. Papageorgiou. Resonant nonlinear Neumann problems with indefinite weight. Ann. Sc. Norm. Super. Pisa Cl. Sci.(5), 11(4):729-788, 2012. https://doi.org/10.2422/2036-2145.201012_003.

[11] N.S. Papageorgiou and V.D. Rădulescu. Coercive and noncoercive nonlinear Neumann problems with indefinite potential. Forum Math., 28(3):545-571, 2016. https://doi.org/10.1515/forum-2014-0094.

[12] N.S. Papageorgiou and V.D. Rădulescu. Nonlinear nonhomogeneous Robin problems with superlinear reaction term. Adv. Nonlinear. Stud., 16(4):737-764, 2016. https://doi.org/10.1515/ans-2016-0023.

[13] N.S. Papageorgiou, V.D. Rădulescu and D.D. Repovš. Positive solutions for perturbations of the Robin eigenvalue problem plus an indefinite potential. Discrete Contin. Dyn. Syst. - A, 37(5):2589-2618, 2017. https://doi.org/10.3934/dcds.2017111.

[14] N.S. Papageorgiou, V.D. Rădulescu and D.D. Repovš. Double-phase problems with reaction of arbitrary growth. Z. Angew. Math. Phys., 69(108), 2018. https://doi.org/10.1007/s00033-018-1001-2.

[15] N.S. Papageorgiou, V.D. Rădulescu and D.D. Repovš. Nodal solutions for nonlinear nonhomogeneous Robin problems. Rend. Lincei Mat. Appl., 29(4):721-738, 2018. https://doi.org/10.4171/RLM/831.

[16] N.S. Papageorgiou, V.D. Rădulescu and D.D. Repovš. Nonlinear Analysis Theory and Methods. Springer, Switzerland, 2019. https://doi.org/10.1007/9783-030-03430-6. 
[17] N.S. Papageorgiou and C. Vetro. Superlinear $(p(z), q(z))$ equations. Complex Var. Ellip. Equ., 64(1):8-25, 2019. https://doi.org/10.1080/17476933.2017.1409743.

[18] N.S. Papageorgiou, C. Vetro and F. Vetro. Multiple nodal solutions for semilinear Robin problems with indefinite linear part and concave terms. Topol. Methods Nonlinear Anal., 50(1):269-286, 2017. https://doi.org/10.12775/TMNA.2017.029.

[19] P. Pucci and J. Serrin. The Maximum Principle. Birkhäuser Verlag, Basel, 2007. https://doi.org/10.1007/978-3-7643-8145-5.

[20] Z.-Q. Wang. Nonlinear boundary value problems with concave nonlinearities near the origin. NoDEA Nonlinear Differential Equations Appl., 8(1):15-33, 2001. https://doi.org/10.1007/PL00001436.

[21] V.V. Zhikov. Averaging of functionals of the calculus of variations and elasticity theory. Math. USSR Izv., 29(1):33-66, 1987. https://doi.org/10.1070/IM1987v029n01ABEH000958.

[22] V.V. Zhikov. On variational problems and nonlinear elliptic equations with nonstandard growth conditions. J. Math. Sci. (N.Y.), 173(5):463-570, 2011. https://doi.org/10.1007/s10958-011-0260-7. 